\title{
Gene transfer during surgical procedures with molecular surgical suture
}

\author{
Dan Huang, ${ }^{1}$ Fan Yu, ${ }^{2}$ Li Jingrong ${ }^{1}$ \\ ${ }^{1}$ Laboratory of Cancer Biology, Hubei Medical University, Hubei, P.R. China. ${ }^{2}$ Laboratory of Molecular \\ Biology, Wuhan University, Wuhan, P.R. China
}

i

(C)2003, European Journal of Histochemistry

Over the last decades, there has been an explosion of interest in plasmid DNA for gene therapy with reports of their efficacy in the fight against cancer, vascular diseases, and inherited diseases caused by specific gene defects (Srivastava, 2003). DNA plasmids present several advantages over the use of recombinant viruses concerning their production and safety issues. Plasmid DNA vectors can be constructed easily and economically, and they are free of size constraints imposed by viral packaging, obviating the need for an infectious vector and lessening the likelihood of toxicity and immunogenicity (Davis, 1993). Plasmids have a relative low cost, long shelf life and allow repetitive administration of the therapeutic gene without generating an immune response against the delivery vector (Donnelly, 2003). Finally, plasmids can be injected directly into tissues, such as heart (Sarkar, 2002), muscle (Neumeister, 2001, Dan, 2000) and tumors (De Marco, 2003, Sasaki, 2002).

Correspondence: Dan Huang, Ph.D., Department of Pathology, Virginia Commonwealth University, Richmond, VA, USA. E-mail: drhuang100@hotmail.com

Key words: gene transfer, suture, muscle.

Paper accepted on December 12, 2003

European Journal of Histochemistry

2004; vol. 48 issue 2 [Apr-Jun]: 111-114
T he potential of in vivo gene therapy and DNA immunization depends upon the development of safe, effective, and economic delivery systems that meet with clinical and patient acceptance. Although there are many systems of DNA delivery into cell, the major disadvantage is their limited clinical utility because of complex and expensive manipulation. With clinical applications in mind, especially for most surgical operation, we tested the effectiveness of surgical suture that is more clinic related, more patient friendly, and more versatile than other delivery methods. To evaluate the potential effectiveness of this approach in human, we used the commonly used surgical suture for DNA delivery into rabbit models first in this study. Overall, we sought to evaluate the effects of gene delivery by means of surgical suture, in light of potential medical applications for gene therapy and DNA vaccinations.

\section{Materials and Methods}

\section{Plasmids construction}

For the construction of pBLacZ, a Hind III-Sst I fragment from the lac $Z$ expression clone was served as the vector. This fragment contained the plasmid sequences for propagation of the clone in E.coli, the SV40 early-region promoter as well as the $3^{\prime}$ end of lacZ followed by the SV40 polyadenylation signal. Downstream from the SV40 promoter carries a HindIII-NcoI fragment carrying the $5^{\prime}$ untranslated region including the eukaryotic ATG start codon that is contained in the NcoI-site. In frame with the start codon, lacZ coding sequences were inserted from the Lac Z expression cassette. Both plasmids were prepared using standard procedures.

Molecular surgical suture preparation for gene transfer The purified pBLacZ plasmid (OD260/0D280= 1.9) was dissolved in PBS buffer with $5 \%$ sucrose $(\mathrm{pH}=7.4)$ and the final concentration was $1 \mu \mathrm{g} / \mathrm{L}$. Surgical suture of $1 \mathrm{~cm}$ length was specifically 
selected. The molecular surgical suture was prepared by soaking suture into the above solution (experimental group) and the PBS solution (control group) overnight and then air dried until the needed amount of plasmids were thoroughly absorbed.

\section{Animal model}

New Zealand white rabbits were used for all experiments. The rabbits (mean weight, $3 \mathrm{~kg}$ ) were anesthetized with ketamine $(50 \mathrm{mg} / \mathrm{kg}$ ) and xylazine (5 $\mathrm{mg} / \mathrm{kg}$ ). Muscle regeneration was induced by injection of $500 \mu \mathrm{L}$ of $0.5 \%$ bupivacaine solution 3 days before plasmid delivery. All rabbits were humanely euthanized by $\mathrm{CO} 2$ asphyxiation at the end of the experiments. These studies were approved by the Institutional Animal Care and Use Committees (IACUC) and performed in accordance with the Guide for the Care and Use of Laboratory Animals.

\section{Intramuscular gene transfer}

5-0 thread was introduced into the anterior tongue of rabbit and the whole tongue was extracted by pulling out of the oral cavity. An incision was cut with a length of $1.5 \mathrm{~cm}$ and a depth of $1 \mathrm{~cm}$ along the lateral of tongue, where blood supply was ample and a surgical assistant was necessary for hemostasis. Intramuscular gene delivery was performed by placing the molecular surgical suture into the incision along the longitudinal axis of the muscle and parallel to the muscle fibers as soon as possible. The incision was quickly closed and marked with a ligature at each end of it. A control piece of muscle was also marked at an area far from this incision site. To keep variability to a minimum, the same skilled operator and assistant performed all procedure after training. The frequence of surgical transfer failure is zero.

\section{In vivo $\beta$-galactosidase histochemistry assay}

Rabbits were sacrificed on the $7^{\text {th }}, 30^{\text {th }}$ days after transfection of lacZ gene. The biopsy of tongue muscle containing the molecular surgical suture between two marking ligatures was removed with a small sharp surgical knife so that the long axis of the strip was parallel to the long axis of the muscle fiber and embedded surgical suture. A control piece of muscle was removed at an area far from this incision site. The serial sections $(5 \mu \mathrm{m})$ made with a cryostat microtome were placed immediately on gelatinized slides. The sections were fixed with $1.25 \%$ glutaraldehyde for $10 \mathrm{~min}$, then rinsed three times with
PBS, and incubated with $\mathrm{X}$-gal staining solution at $37^{\circ} \mathrm{C}$ for 48 hours. After incubation, sections were rinsed with PBS and then cleared in increasing concentrations of alcohol and xylene, and mounted with coverslips in mounting medium.

\section{RT-PCR}

Total RNA from transfected muscles and control tissues was extracted with TRIZOL reagent (Gibco$B R L, N Y$, USA) following the manufacturer's instruction. The RNA was reverse-transcribed using the SuperScriptTM Preamplification System (Gibco-BRL, MD, USA), and the resulting CDNA was amplified by PCR using Taq DNA polymerase (Takara Shuzou, Shiga, Japan) and the specific primers for LacZ. The following oligonucleotides were used as primers for PCR: the LacZ sense primer 5-CAATCCCCTGTGGAGGCTCCA-3 and the anti-sense primer 5-GACCAAGTCGTCACCTCGCAG-3. Samples were subjected to 28 cycles of $1 \mathrm{~min}$ of denaturation at $94^{\circ} \mathrm{C}, 1 \mathrm{~min}$ of annealing at $61^{\circ} \mathrm{C}$ and $1 \mathrm{~min}$ of synthesis at $72^{\circ} \mathrm{C}$. The gene expression level of RT-PCR bands was measured by densitometer.

\section{Results}

\section{Expression of $\beta$-galactosidase protein}

$\beta$-galactosidase as marker enzyme provides the advantage that the location of expressed foreign DNA can be readily determined. Expression of $\beta$ galactosidase protein in the myofibers of tongue muscle was observed in the vicinity of the suture seven days and one month after the gene transfer, no control muscle contained any positive myofibers (Figure 1). These results indicate that non-human rodent myofibers have the ability to uptake and express the pasmid DNA by molecular surgical suture.

\section{Expression of $\beta$-galactosidase $\mathbf{m R N A}$}

$\beta$-galactosidase mRNA was positive in PBLacZ transfected tongue muscle one week and one month after gene transfer by molecular surgical suture. RTPCR for $\beta$-galactosidase mRNA in control group was negative (Figure 2).

\section{Discussion}

In this study, we investigated the pattern of plasmid DNA transfer by surgical suture that are suit- 


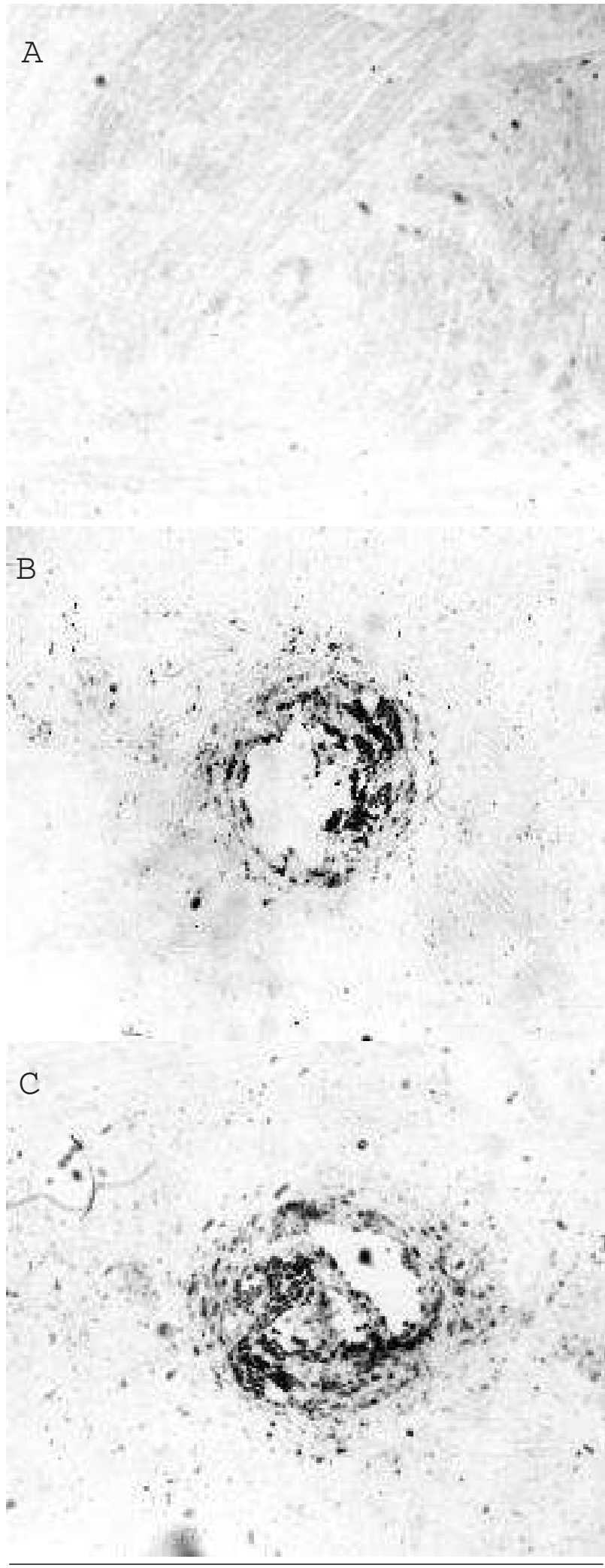

Figure 1. Intramuscular gene transfer of rabbit tongue muscle transfected with pBLacZ plasmid encoding nuclear localized $\beta$ Gal. a. Section of control group showing no positive cells. b. Expression of $\beta$-galactosidase protein in the myofibers of tongue muscle was observed in the vicinity of the suture seven days after the gene transfer. c. 30 days after the gene transfer in experimental group. able for clinical applications. We also showed, for the first time, the effectiveness of DNA delivery into tongue muscle on rabbits by surgical suture, which might give suggestion of potential applicability of this method on human gene therapy and DNA vaccination.

The new technique for gene transfer in vivo described in this report conforms to the method used clinically and is compatible with the most clinical surgical operation procedures. In this regard, effective gene can be clinically incubated in surgical area by surgical suture during operation procedure because we must handle with muscles in most of surgical operations. As a delivery system, the absorbable cotton suture might act not only as gene carrier, but also as an adjuvant that could produce a cushioning effect on the uptake of high doses of plasmid through slow release of the absorbed plasmid DNA into the interstitial space (Labhasetwar, 1998). Thus it prevented the rapid degradation of DNA following injection and promoted their access to myofibers. In this report, tongue muscles are composed of large skeletal muscle fiber cells containing hundreds of postmitotic myonuclei, which might account for the high efficiency of plamid DNA uptake although the mechanisms are not precisely known. This interpretation is supported by histological and RT-PCR evidences showing that lacZ expression indeed takes place primarily in the muscle layer under the experimental conditions used.

As far as the biosafety of the current method is concerned, no signs of adverse effects or irreversible alteration of function were detected during our experiment, and no impairment of skin, hair, necrosis were observed in any of the rabbits. However, tongue edema was observed in the animals after gene transfer, and the edema diminished in a couple of days. In

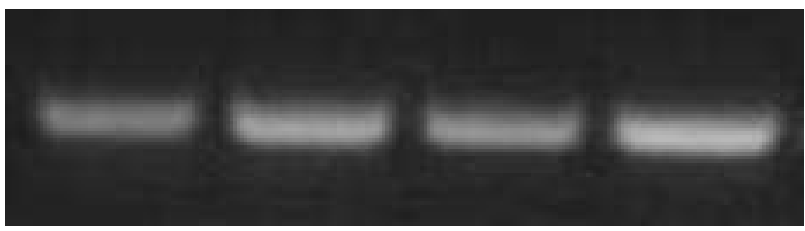

Figure 2. RT-PCR analysis of the pBLacZ transfected muscle. From the left: 1. Blank; 2. Control group; 3 . Tongue muscles 7 days after the gene transfer (DV:28); 4. Tongue muscles 30 days after the gene transfer (DV:12). The densitometric value (DV) is the measurement of the gene expression level of RT-PCR bands by densitometer. 
one pre-experimental rabbit, tongue edema was so massive that the animal had to be sacrificed 9 days after the gene transfer. This kind of abnormality was no longer observed in the other experimental animals. Some recent researches have provided encouraging human immune response data in support of this plasmid DNA transfer technology (Conry, 2002, Huang, 2001). The next step should be the clinical assessment of the safety and efficacy of plasmid DNA delivery with gene transfer suture if possible.

The findings of the current study are likely to provide a useful gene transfer approach that may be applicable to the surgical procedures for human gene therapy. Further study should be made to solve the problem of efficiency of gene expression (Niidome, 2002). It has been reported that electroporation methods significantly enhance direct injection of naked DNA (Martin, 2000) and in vivo transfection methods with antisense oligonucleotides and small interfering RNA (siRNA) showed greatly increased efficiency of naked DNA gene transfer. (Herweije, 2003). Combined with these new advance and clinical cancer related gene (Dan,2003), our molecular surgical suture technology will make naked DNA gene transfer a viable method for gene therapy in the near future.

\section{References}

Conry RM, Curiel DT, Strong TV, Moore SE, Allen KO, Barlow DL, et al. Safety and immunogenicity of a DNA vaccine encoding carcinoembryonic antigen and hepatitis B surface antigen in colorectal carcinoma patients. Clin Cancer Res 2002;8:2782-7.

Dan Huang, Jingrong Li. The establishment of gene transfer into rabbit sternoclaidomastoid muscle in vivo and optimization. $J$ Head Neck Surg 2000;10:308-11.

Davis HL, Whalen RG, Demeneix BA. Direct gene transfer into skeletal muscle in vivo: factors affecting efficiency of transfer and stability of expression. Hum Gene Ther 1993;4:151-9.

Dan Huang, Wantao Chen, Ronggen He. Identification of genes with consistent expression alteration pattern in ACC-2 and ACC-M cells by cDNA array. Chin Med J 2003;116:448-52.

De Marco F, Hallez S, Brulet JM, Gesche F, Marzano P, Flamini S, et al. DNA vaccines against HPV-16 E7-expressing tumour cells. Anticancer Res 2003;23:1449-54.

Donnelly J, Berry K, UImer JB. Technical and regulatory hurdles for DNA vaccines. Int J Parasitol 2003;33:457-67.

Herweijer H, Wolff JA. Progress and prospects: naked DNA gene transfer and therapy. Gene Ther 2003;10:453-8.

Huang Dan, Li Jingrong. The safety of antigenicity gene transfection with surgical suture into rabbit's tongue muscle. J Pract Stomatol 2001; 17:6-8.

Labhasetwar V, Bonadio J, Goldstein S, Chen W, Levy RJ. A DNA controlled-release coating for gene transfer: transfection in skeletal and cardiac muscle. J Pharm Sci 1998;87:1347-50

Martin JB, Young JL, Benoit JN, Dean DA. Gene transfer to intact mesenteric arteries by electroporation. J Vasc Res 2000;37:372-80

Neumeister MW, Song YH, Mowlavi A, Suchy H, Mathur A. Effects of liposome-mediated gene transfer of VEGF in ischemic rat gracilis muscle. Microsurgery 2001;21:58-62

Niidome T, Huang L. Gene therapy progress and prospects: nonviral vectors. Gene Ther 2002;9:1647-52.

Sarkar N, Blomberg P, Wardell E, Eskandarpour M, Sylven C, Drvota $\checkmark$, et al. Nonsurgical direct delivery of plasmid DNA into rat heart: time course, dose response, and the influence of different promoters on gene expression. J Cardiovasc Pharmacol 2002;39:215-24

Sasaki Y, Kasuya K, Nishihira J, Magami Y, Tsuchida A, Aoki T, et al. Suppression of tumor growth through introduction of an antisense plasmid of macrophage migration inhibitory factor. Int $\mathrm{J}$ Mol Med 2002;10:579-83.

Srivastava IK, Liu MA. Gene vaccines. Ann Intern Med 2003;138: $550-9$ 